\title{
SATURATION OF THE MASSIVE SCHWINGER MODEL
}

\author{
H.O. GIROTTI ${ }^{1}$ \\ Instituto de Física, Universidade Federal do Rio Grande do Sul, \\ 90000 Porto Alegre, RS, Brazil
}

Received 28 September 1979

\begin{abstract}
We determine the field configurations saturating the massive Schwinger model (MSM). Fermion confinement is proved, for the MSM, without invoking the Bose form of the theory. A gas of induced instantons accounts for the presence of the bare fermion mass.
\end{abstract}

In this note we present the modifications induced by a small, but finite, bare fermion mass on the field configurations saturating the fermion propagator in the massless Schwinger model (SM) [1-4] .

We show that the c-instanton (half integer winding number) [3] emerges in each order of mass perturbation theory. Thus, we prove fermion confinement in the massive Schwinger model (MSM) without invoking the Bose form of the theory [5-7] . A similar conclusion applies for the vacuum structure exhibited by the MSM.

Bosonization of fermions is a concept arising from the absence of intrinsic spin in a two-dimensional spacetime and, as such, it cannot be boosted into a four-dimensional world. Therefore, our proof for fermion confinement might be of some utility when the MSM is used to mimic some situations encountered in the four-dimensional theory describing the strong interactions [8], the essential point being, of course, that the addition of a small bare mass, to the otherwise massless quark fields, should not destroy the confinement mechanism.

Moreover, we show that the mass effects can be globally rephrased into topological language. By this we mean that a gas of induced instantous (integer winding number) accounts for the presence of a nonvanishing bare fermion mass. This statement also admits "translation" into a four-dimensional space-time.

The MSM is defined by the lagrangian density

$\varrho=-\frac{1}{4} F_{\mu \nu} F^{\mu \nu}+\bar{\psi}\left[\mathrm{i} \gamma^{\mu}\left(\partial_{\mu}-\mathrm{i} e A_{\mu}\right)\right] \psi$,

where $\psi$ is a two-component massive fermion field (bare mass $m$ and charge $e$ ), $A_{\mu}$ is the electromagnetic field, and $F_{\mu \nu}$ is its corresponding field strength tensor, i.e., $F_{\mu \nu}=\partial_{\mu} A_{\nu}-\partial_{\nu} A_{\mu}$. Our conventions and notations are those of ref. [4]. The model is quantized in an arbitrary covariant gauge parametrized by $\alpha$ ( $\alpha=0$ corresponds to Schwinger's [9] original gauge).

We are interested in the fermion propagator $\left\langle\psi(x) \bar{\psi}\left(x^{\prime}\right)\right\rangle$. Since the MSM is not an exactly solvable theory we look for a solution of the form ( $a$ and $b$ are spin indices)

$\left\langle\psi_{a}(x) \bar{\psi}_{b}\left(x^{\prime}\right)\right\rangle=\sum_{k=0}^{\infty} m^{k}\left\langle\psi_{a}(x) \bar{\psi}_{b}\left(x^{\prime}\right)\right\rangle_{k}$.

This expansion is assumed to converge, for fixed $e$, in a finite neighborhood of $m=0$. Consequently, we can only assure the validity of our results for the so-called strong coupling regime of the MSM [6], i.e. for $e / m \gg 1$.

The power series representation in (2) essentially implies that we are expanding the MSM "around" the SM. In

${ }^{1}$ Supported in part by Conselho Nacional de Desenvolvimento Científico e Tecnológico (CNPq), Brazil. 
fact, one can demonstrate that, after carrying out the functional integrations in the fermionic degrees of freedom, all coefficient functions in (2) are given in terms of quadratic path integrals over zero-winding configurations of the $A_{\mu}$-field. This allows for an interpretation of the properties of each coefficient function, and, therefore, of the entire propagator, in terms of saturating field configurations. For the first few terms of (2) we obtain

$$
\begin{aligned}
& \left\langle\psi_{1}(x) \bar{\psi}_{1}\left(x^{\prime}\right)\right\rangle_{0}=N \int\left[\mathrm{D} A_{\mu}\right] \exp \left\{\mathrm{i} S_{\mathrm{eff}}^{(0)}\left[A_{\mu} \mid x, x^{\prime}\right]\right\} G_{\mathrm{F}_{11}}\left(x, x^{\prime}\right), \\
& \left\langle\psi_{1}(x) \bar{\psi}_{1}\left(x^{\prime}\right)\right\rangle_{1}=N \int \mathrm{d}^{2} y \int\left[\mathrm{D} A_{\mu}\right] \exp \left\{\mathrm{i} S_{\mathrm{eff}}^{(1)}\left[A_{\mu} \mid x, y, x^{\prime}\right]\right\}\left[G_{\mathrm{F}}(x, y) G_{\mathrm{F}}\left(y, x^{\prime}\right)\right]_{11}, \\
& \left\langle\psi_{1}(x) \bar{\psi}_{1}\left(x^{\prime}\right)\right\rangle_{2}=N \int \mathrm{d}^{2} y_{1} \int \mathrm{d}^{2} y_{2} \int\left[\mathrm{D} A_{\mu}\right]\left\{\exp \left\{\mathrm{i} S_{\mathrm{eff}}^{(2)}\left[A_{\mu} \mid x, y_{1}, y_{2}, x^{\prime}\right]\right\}\left[G_{\mathrm{F}}\left(x, y_{1}\right) G_{\mathrm{F}}\left(y_{1}, y_{2}\right) G_{\mathrm{F}}\left(y_{2}, x^{\prime}\right)\right]_{11}\right. \\
& \left.\quad+\frac{1}{2(2 \pi)^{2}} \frac{1}{\left(y_{1}-y_{2}\right)^{2}}\left[\exp \left\{\mathrm{i} S_{\mathrm{eff}}^{(2)+}\left[A_{\mu} \mid x, y_{1}, y_{2}, x^{\prime}\right]\right\}+\exp \left\{\mathrm{i} S_{\mathrm{eff}}^{(2)-}\left[A_{\mu} \mid x, y_{1}, y_{2}, x^{\prime}\right]\right\}\right] G_{\mathrm{F}_{11}}\left(x, x^{\prime}\right)\right\} .
\end{aligned}
$$

Here, $G_{\mathrm{F}}\left(x, x^{\prime}\right)$ is the free massless fermion Green's function and $N$ is a normalization constant.

To continue this process for the higher-order coefficients of the mass expansion (2) only adds algebraic complications. It is readily seen that the effective action, $S_{\text {eff }}^{(k)}$, corresponding to the coefficient function $\left\langle\psi_{1}(x) \bar{\psi}_{1}\left(x^{\prime}\right)\right\rangle_{k}$ is quadratic in the field variable $A_{\mu}$ and it can be generically written as follows:

$S_{\text {eff }}^{(k)}\left[A_{\mu} \mid x, y_{1}, \ldots, y_{k}, x^{\prime}\right]=\frac{1}{2} \int \mathrm{d}^{2} z \int \mathrm{d}^{2} z^{\prime} A^{\mu}(z) \Omega_{\mu \nu}^{(\alpha)}\left(z, z^{\prime}\right) A^{\nu}\left(z^{\prime}\right)+\int \mathrm{d}^{2} z A^{\mu}(z) \Lambda_{\mu}^{(k)}\left(z ; x, y_{1}, \ldots, y_{k}, x^{\prime}\right)$,

where

$\Omega_{\mu \nu}^{(\alpha)}\left(z, z^{\prime}\right)=\left\{g_{\mu \nu}\left(\square_{z}+e^{2} / \pi\right)-\partial_{z_{\mu}}\left[1-\alpha^{-1}+\left(e^{2} / \pi\right) \square_{z}^{-1}\right] \partial_{z_{\mu}}\right\} \delta^{(2)}\left(z-z^{\prime}\right)$,

and $\Lambda_{\mu}^{(k)}$ is an $A_{\mu}$-independent external-current-like factor.

In particular, one finds that

$\Lambda_{\mu}^{(0)}\left(z ; x, x^{\prime}\right)=\Lambda_{\mu_{\mathrm{L}}}\left(z ; x, x^{\prime}\right)+e \epsilon_{\mu \nu} \partial_{z}^{\nu}\left[D_{\mathrm{F}}(x-z)-D_{\mathrm{F}}\left(x^{\prime}-z\right)\right]$,

$\Lambda_{\mu}^{(1)}\left(z ; x, y, x^{\prime}\right)=\Lambda_{\mu_{\mathrm{L}}}\left(z ; x, x^{\prime}\right)+e \epsilon_{\mu \nu} \partial_{z}^{\nu}\left[D_{\mathrm{F}}(x-z)-2 D_{\mathrm{F}}(y-z)+D_{\mathrm{F}}\left(x^{\prime}-z\right)\right]$,

$\Lambda_{\mu}^{(2)}\left(z ; x, y_{1}, y_{2}, x^{\prime}\right)=\Lambda_{\mu_{\mathrm{L}}}\left(z ; x, x^{\prime}\right)+e \epsilon_{\mu \nu} \partial_{z}^{\nu}\left[D_{\mathrm{F}}(x-z)-2 D_{\mathrm{F}}\left(y_{1}-z\right)+2 D_{\mathrm{F}}\left(y_{2}-z\right)-D_{\mathrm{F}}\left(x^{\prime}-z\right)\right]$,

$\Lambda_{\mu}^{(2) \pm}\left(z ; x, y_{1}, y_{2}, x^{\prime}\right)=\Lambda_{\mu \mathrm{L}}\left(z ; x, x^{\prime}\right)+e \epsilon_{\mu \nu} \partial_{z}^{\nu}\left[D_{\mathrm{F}}(x-z) \mp 2 D_{\mathrm{F}}\left(y_{1}-z\right) \pm 2 D_{\mathrm{F}}\left(y_{2}-z\right)-D_{\mathrm{F}}\left(x^{\prime}-z\right)\right]$,

where $D_{\mathrm{F}}\left(x-x^{\prime}\right)\left[\Delta_{\mathrm{F}}\left(x-x^{\prime} ; m^{2}\right)\right]$ designates the massless [massive] free propagator for spinless particles. The infrared divergences in $D_{\mathrm{F}}$ are avoided by the introduction of an infrared regulator mass $\mu$. Since a finite renormalization will not alter our conclusions, we choose $\mu=e / \sqrt{\pi}$. The longitudinal part of $\Lambda_{\mu}^{(k)}$ is given by

$\Lambda_{\mu_{\mathrm{L}}}\left(z ; x, x^{\prime}\right)=-e \partial_{z_{\mu}}\left[D_{\mathrm{F}}(x-z)-D_{\mathrm{F}}\left(x^{\prime}-z\right)\right]$.

We remark that $\Lambda_{\mu}^{(2)}, \Lambda_{\mu}^{(2)+}$ and $\Lambda_{\mu}^{(2)-}$ possess a similar structure. The contributions to $\left\langle\psi_{1}(x) \bar{\psi}_{1}\left(x^{\prime}\right)\right\rangle_{2}$ coming from the functional fermion determinant are contained in $\Lambda_{\mu}^{(2) \pm}$, while $\Lambda_{\mu}^{(2)}$ arises from the corrections of order $m^{2}$ to the fermion Green's function in an external field $A_{\mu}$. Since terms linear in the fermion mass are not present in the mass expansion of the functional fermion determinant, this determinant does not contribute to $\left\langle\psi_{1}(x)\right.$ $\left.X \bar{\psi}_{1}\left(x^{\prime}\right)\right\rangle_{1}$.

We turn now into the problem of finding the field configurations, $\widetilde{A}_{\mu}$, saturating the functional integrals appear- 
ing in eqs. (3)-(5). They follow from $[2-4,10]$

$\delta S_{\text {eff }}\left[A_{\mu} \mid x, \ldots, x^{\prime}\right] /\left.\delta A_{\mu}(z)\right|_{A_{\mu}=\tilde{A}_{\mu}}=0$,

and read, respectively,

$\widetilde{A}_{\mu}^{(0)}\left(z ; x, x^{\prime}\right)=R_{\mu}^{-\cdot}(z, x)+R_{\mu}^{+}\left(z, x^{\prime}\right)$,

$\widetilde{A}_{\mu}^{(1)}\left(z ; x, y, x^{\prime}\right)=R_{\mu}^{-}(z, x)+2 R_{\mu}^{+}(z, y)+R_{\mu}^{-}\left(z, x^{\prime}\right)$,

$\widetilde{A}_{\mu}^{(2)}\left(z ; x, y_{1}, y_{2}, x^{\prime}\right)=R_{\mu}^{-}(z, x)+2 R_{\mu}^{+}\left(z, y_{1}\right)+2 R_{\mu}^{-}\left(z, y_{2}\right)+R_{\mu}^{+}\left(z, x^{\prime}\right)$,

$\widetilde{A}_{\mu}^{(2) \pm}\left(z ; x, y_{1}, y_{2}, x^{\prime}\right)=R_{\mu}^{-}(z, x)+2 R_{\mu}^{ \pm}\left(z, y_{1}\right)+2 R_{\mu}^{\mp}\left(z, y_{2}\right)+R_{\mu}^{+}\left(z, x^{\prime}\right)$.

Here

$R_{\mu}^{ \pm}(z, x)=\mp(\pi / e) \epsilon_{\mu \nu} \partial_{z}^{\nu}\left[\Delta_{\mathrm{F}}\left(z-x ; e^{2} / \pi\right)-D_{\mathrm{F}}(z-x)\right]$,

and a longitudinal (gauge-dependent) part has been systematically omitted because it does not contribute to the winding number. Needless to say, $\widetilde{A}_{\mu}^{(0)}$ is the configuration found by Nielsen and Schroer [3] for the SM. It describes a sum of a c-instanton $\left(R_{\mu}^{+}\right)$and a c-antiinstanton $\left(R_{\mu}^{-}\right)$, the corresponding winding numbers being equal to $+\frac{1}{2}$ and $-\frac{1}{2}$, respectively. On the other hand, $2 R_{\mu}^{+}\left(2 R_{\mu}^{-}\right)$is a configuration with winding number equal to $+1(-1)$; therefore, we shall refer to it as an instanton (antiinstanton).

The generalization of the results presented in eqs. (14)-(17) lead us to the conclusion that the generic $k$ th coefficient function in (2) is saturated by a field configuration $\widetilde{A}_{\mu}^{(k)}$ describing the sum of two c-instantons, attached to the external points $\left(x, x^{\prime}\right)$, plus $k$ instantons, which are peaked around the internal points $\left(y_{1}, \ldots, y_{k}\right)$. Of course, the configuration, as a whole, must be "topologically neutral".

Then, the appearance of an instanton gas is one of the main effects resulting from the addition of a bare mass to the fermion field.

Furthermore, the presence of the c-instanton, in the field configuration saturating each term in the expansion (2), is conclusive evidence that, in the MSM, fermions remain confined. Also, there must exist a vacuum structure in which two successive vacua are connected by gauge transformations with a class index equal to $1 / 2$. These conclusions are known to be true $[3,7,11]$ but, in the present work, we have arrived at them through a perturbative approach and without invoking the bosonized form of the theory.

The possibility that an instanton gas might account for the presence of a nonvanishing bare fermion mass was suggested to us by Professor J.A. Swieca, to whom we are indebted.

\section{References}

[1] N.K. Nielsen and B. Schroer, Nucl. Phys. B120 (1977) 62.

[2] N.K. Nielsen and B. Schroer, Phys. Lett. 66B (1977) 73.

[3] N.K. Nielsen and B. Schroer, Phys. Lett. 66B (1977) 475.

[4] H.O. Girotti, Phys. Rev. D19 (1979) 724.

[5] S. Coleman, R. Jackiw and L. Susskind, Ann. Phys. 93 (1975) 267.

[6] S. Coleman, Ann. Phys. 101 (1976) 239.

[7] K.D. Rothe and J.A. Swieca, Phys. Rev. D15 (1978) 1675.

[8] J. Kogut and L. Susskind, Phys. Rev. D11 (1975) 3594.

[9] J. Schwinger, Phys. Rev, 128 (1962) 2425.

[10] H.O. Girotti, Nuovo Cimento Lett. 24 (1979) 233.

[11] K.D. Rothe and J.A. Swieca, Phys. Rev. D1 5 (1977) 541. 